\title{
EFEITO DA TEMPERATURA DA FERMENTAÇÃO MALOLÁTICA NA COMPOSIÇÃO FENÓLICA DO VINHO 'CABERNET SAUVIGNON' EM REGIÃO DE ELEVADA ALTITUDE DE SANTA CATARINA
}

\author{
${ }^{1}$ Douglas André Wurz, ${ }^{2}$ Juliana Reinher, ${ }^{3}$ Adrielen Tamiris Canossa, ${ }^{4}$ Leo Rufato, ${ }^{5}$ Ricardo Allebrandt, \\ ${ }^{6}$ Betina Pereira de Bem, ${ }^{7}$ Alberto Fontanella Brighenti

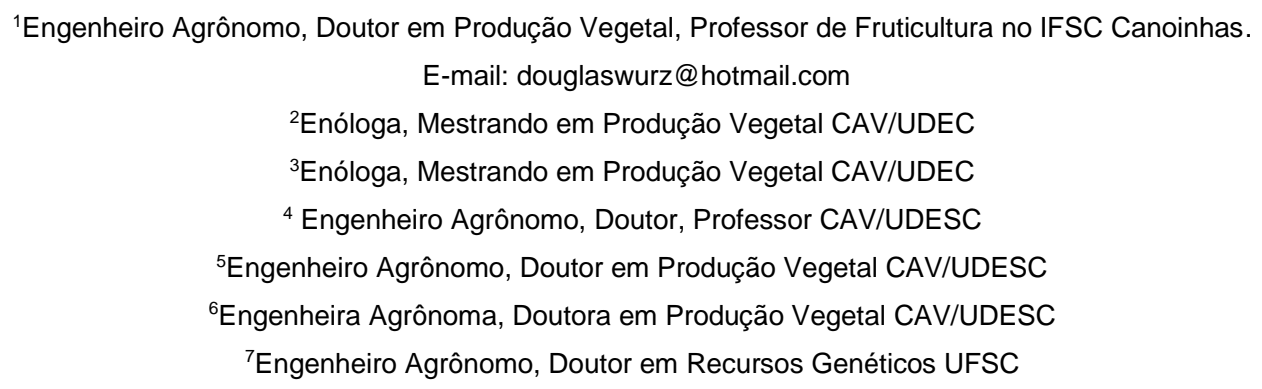

RESUMO: O objetivo deste trabalho foi avaliar o efeito de diferentes temperaturas durante a fermentação malolática espontânea e as características químicas do vinho 'Cabernet Sauvignon', proveniente de região de elevada altitude de Santa Catarina, Brasil. O presente trabalho foi realizado no Laboratório de Enologia da Universidade do Estado de Santa Catarina. A uva foi proveniente do vinhedo da Estação Experimental de São Joaquim. As garrafas contendo os vinhos elaborados foram condicionadas em BOD's e submetidas à diferentes temperaturas para realização da fermentação malolática: $10^{\circ} \mathrm{C}, 20^{\circ} \mathrm{C}, 30^{\circ} \mathrm{C}, 40^{\circ} \mathrm{C}$ e $60^{\circ} \mathrm{C}$. Em cada coleta foram avaliados os teores de antocianinas, polifenóis totais e a coloração dos vinhos. A temperatura da fermentação malolática tem influência na composição química do vinho Cabernet Sauvignon, sendo que o aumento da temperatura reduz o teor de antocianinas, além de reduzir a coloração vermelha do vinho, representando pelo aumento da tonalidade de coloração dos vinhos. A temperatura da fermentação malolática não influenciou o conteúdo de polifenóis totais do vinho Cabernet Sauvignon. E a conclusão?

Palavras-chave: Vitis vinifera L., vitivinicultura, enologia, polifenóis, antocianinas, intensidade de cor.

\section{EFFECT OF MALOLACTIC FERMENTATION TEMPERATURE ON THE PHENOLIC COMPOSITION OF 'CABERNET SAUVIGNON' WINE IN HIGH ALTITUDE REGIONS OF SANTA CATARINA STATE}


ABSTRACT: The objective of this work was to evaluate the effect of different temperatures of the malolactic fermentation and the effect on the chemical characteristics of 'Cabernet Sauvignon' grapevine from high altitude regions of Santa Catarina State, Brazil. The present work was carried out in the Oenology Laboratory of the State University of Santa Catarina State. The grapes came from the vineyard of the São Joaquim Experimental Station (EPAGRI). For the elaboration of the wine were used $80 \mathrm{~kg}$ of 'Cabernet Sauvignon' grapevines. The bottles were placed in BOD's and submitted to different temperatures for malolactic fermentation: $10^{\circ} \mathrm{C}, 20^{\circ} \mathrm{C}, 30^{\circ} \mathrm{C}$, $40^{\circ} \mathrm{C}$ and $60^{\circ} \mathrm{C}$. In each collection, the anthocyanins, total polyphenols and the color of the wines were evaluated. The data were submitted to analysis of variance and compared by the Tukey Test at 5\% probability of error. The temperature of the malolactic fermentation has an influence on the chemical composition of the 'Cabernet Sauvignon' wine. The increase in temperature reduces anthocyanin content, as well as reducing the red color of the wine, representing an increase in the color of wines. The temperature of the malolactic fermentation did not influence the total polyphenol content of 'Cabernet Sauvignon' wine.

Key words: Vitis vinifera L., viticulture, enology, polyphenols, anthocyanins, color intensity.

\section{INTRODUÇÃO}

A região do Planalto Sul Catarinense brasileiro vem se destacando na elaboração de vinhos finos de qualidade. Devido às condições particulares da região, como elevada altitude (acima de 1000 metros do nível do mar) e condições climáticas que permitem um ciclo vegetativo mais longo das videiras, há o alcance de índices de maturação que fornecem matéria prima para a elaboração de vinhos diferenciados por sua intensa coloração, definição aromática e equilíbrio gustativo (BORGHEZAN et al., 2014; MALINOVSKI et al., 2016; WURZ et al., 2017).

Um dos principais problemas encontrados pelos viticultores e enólogos da região de altitude de Santa Catarina, na fase de elaboração dos vinhos é a dificuldade do término da fermentação malolática. Esta fermentação é desejável, principalmente em vinhos tintos de regiões frias com alto teor residual de ácidos orgânicos, pois é responsável pela descarboxilação do ácido málico e sua transformação em ácido láctico, o qual é menos agressivo e mais aceitável ao paladar humano (JACKSON, 2014). O vinho é um meio favorável para o crescimento microbiano, composição, e a 
temperatura de fermentação podem contribuir para alterações na fermentação malolática (MOTA et al., 2018).

A fermentação malolática ocorre tradicionalmente de forma espontânea, realizada pela microbiota natural presente nas folhas da videira, solo, cachos de uva e nos equipamentos da vinícola. Todavia, existem alguns riscos inerentes a essa abordagem, como a demora excessiva para finalização do processo ou mesmo a não realização dessa fermentação. Isso pode ocorrer devido à condições muito estressantes no vinho para crescimento adequado das bactérias, principalmente temperaturas inadequadas ou uso de produtos químicos como tratamentos sanitizantes durante a preparação do mosto (BINATTI, 2015). A temperatura para o desenvolvimento destes microorganismos deve ser de $15^{\circ} \mathrm{C}$ a $18^{\circ} \mathrm{C}$, e, uma vez iniciada, ela continuará, mesmo se a temperatura for inferior a $15^{\circ} \mathrm{C}$, porém, abaixo de $12^{\circ} \mathrm{C}$, a fermentação torna-se demorada, correndo, inclusive, o risco de ser interrompida (RIZZON; MANFROI, 2006).

O objetivo deste trabalho foi avaliar o efeito de diferentes temperaturas sobre a fermentação malolática espontânea e as características químicas do vinho 'Cabernet Sauvignon', proveniente de região de elevada altitude de Santa Catarina.

\section{MATERIAL E MÉTODOS}

O presente trabalho foi realizado no Laboratório de Enologia da Universidade do Estado de Santa Catarina (CAV/UDESC), Lages, Santa Catarina (SC). A uva foi proveniente do vinhedo da Empresa de Pesquisa Agropecuária e Extensão Rural de Santa Catarina - Estação Experimental de São Joaquim (EPAGRI - EESJ; 28¹7'39 "S, 4955'56" W, altitude $1.415 \mathrm{~m}$ ), safra 2016/2017.

Para elaboração do vinho foram utilizados $80 \mathrm{~kg}$ de uva 'Cabernet Sauvignon' e as microvinificações foram realizadas conforme o protocolo adaptado de Pszczolkowski; Lecco (2011) e Makhotkina et al. (2013).

Após o término da fermentação alcoólica, foram coletadas 25 amostras em garrafas de $375 \mathrm{~mL}$, nas quais foram adaptados batoques hidráulicos. As garrafas foram colocadas em BOD's e submetidas à diferentes temperaturas para a realização da fermentação malolática: $10^{\circ} \mathrm{C}, 20^{\circ} \mathrm{C}, 30^{\circ} \mathrm{C}, 40^{\circ} \mathrm{C}$ e $60^{\circ} \mathrm{C}$, com cinco garrafas por repetição. Foram realizadas cinco coletas dos vinhos sob as diferentes temperaturas 
ao longo do período de fermentação. Em cada coleta foram avaliados os teores de antocianinas, polifenóis totais e a coloração dos vinhos.

A concentração de polifenóis totais (PT) na casca foi determinada pelo método de espectrofotometria, descrito por Singleton; Rossi (1965), utilizando o reagente Folin-Ciocalteu (Vetec) e o ácido gálico como padrão, com leituras da absorbância em $760 \mathrm{~nm}$. O teor de antocianinas na casca e a coloração dos vinhos foram determinadas pelo método de espectrofotometria, descrito por Rizzon (2010). O extrato foi diluído na proporção 1:10 e analisado em espectrofotômetro nos comprimentos de onda de $420 \mathrm{~nm}, 520 \mathrm{~nm}$ e $620 \mathrm{~nm}$. A cor foi mensurada pelos parâmetros de intensidade e tonalidade de cor, obtida através das fórmulas: Intensidade $=420+520+620 \mathrm{~nm}$ e Tonalidade $=420 / 520 \mathrm{~nm}$. Para o acompanhamento da fermentação malolática foram realizadas análises de cromatografia de papel para detectar a presença ou ausência de ácido málico.

O delineamento experimental utilizado foi inteiramente casualizado, com cinco repetições por tratamento. Os dados foram submetidos à análise de variância (ANOVA) e comparados pelo Teste Tukey a $5 \%$ de probabilidade de erro.

\section{RESULTADOS}

As diferentes temperaturas da fermentação malolática influenciaram a composição química do vinho 'Cabernet Sauvignon', conforme descrito na Tabela 1.

Observou-se que o aumento da temperatura da fermentação malolática reduziu o conteúdo de antocianinas totais no vinho. Não se observou efeito das diferentes temperaturas no conteúdo de polifenóis totais no vinho.

Tabela 1 - Efeito de diferentes temperaturas de fermentação malolática nas propriedades químicas do vinho da uva 'Cabernet Sauvignon' proveniente de região de elevada altitude de Santa Catarina,, safra 2016/2017.

Table 1 - Effect of different temperatures of malolactic fermentation on the properties of 'Cabernet Sauvignon' grape from high altitude regions of Santa Catarina, Lages / SC, 2016/2017 harvest.

\begin{tabular}{|c|c|c|c|c|}
\hline $\begin{array}{l}\text { Temperatura } \\
\text { Fermentação }\end{array}$ & $\begin{array}{c}\text { Antocianinas } \\
\left(\mathbf{m g} \cdot \mathrm{L}^{-1}\right)\end{array}$ & $\begin{array}{c}\text { Polifenóis Totais } \\
\left(\mathrm{mg} \cdot \mathrm{L}^{-1}\right)\end{array}$ & $\begin{array}{c}\text { Intensidade de } \\
\text { Cor } \\
(420+520+620 \\
\mathrm{nm}) \\
\end{array}$ & $\begin{array}{c}\text { Tonalidade } \\
\text { de Cor } \\
(420 / 520 \mathrm{n} \\
\mathrm{m}) \\
\end{array}$ \\
\hline $10^{\circ} \mathrm{C}$ & $830.3 a$ & $2561.2 \mathrm{~ns}$ & $13.4 \mathrm{~b}$ & $0.67 \mathrm{c}$ \\
\hline $20^{\circ} \mathrm{C}$ & $554.8 \mathrm{~b}$ & 2274.4 & $12.5 \mathrm{~b}$ & $0.76 \mathrm{c}$ \\
\hline $30^{\circ} \mathrm{C}$ & $236.7 c$ & 2709.3 & $16.0 \mathrm{a}$ & $0.90 \mathrm{~b}$ \\
\hline $40^{\circ} \mathrm{C}$ & $151.3 \mathrm{c}$ & 2348.4 & $17.1 \mathrm{a}$ & $1.02 \mathrm{~b}$ \\
\hline
\end{tabular}




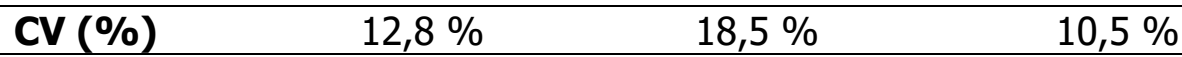

*Médias seguidas pela mesma letra, na coluna, não diferem significativamente entre si, pelo teste de Scott Knott a $5 \%$.

ns = não significativo pela análise de variância (ANOVA) a 5\% de probabilidade de erro.

A intensidade e a tonalidade de cor do vinho da uva 'Cabernet Sauvignon' apresentou valores superiores com o aumento da temperatura malolática, no entanto, ressalta-se que os valores observados apresentam efeito negativo na qualidade do vinho, pois o aumento da intensidade e da tonalidade de coloração dos vinhos no presente trabalho estão associados a redução da coloração vermelho/violáceo dos vinhos, conforme pode ser observado na Figura 1. As temperaturas de fermentação malolática de $10^{\circ} \mathrm{C}$ e $20^{\circ} \mathrm{C}$ foram as que não apresentaram redução das variáveis tonalidade e intensidade de coloração (Tabela 1).

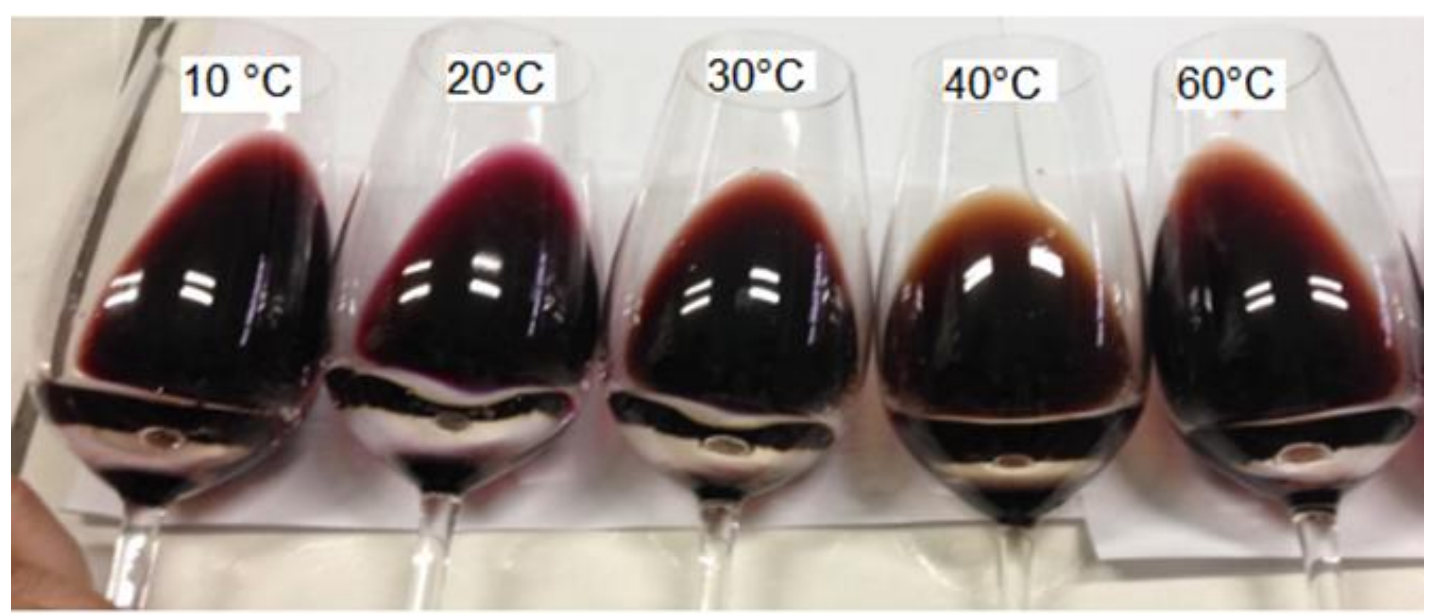

Figura 1 - Efeito de diferentes temperaturas de fermentação malolática na coloração do vinho da uva 'Cabernet Sauvignon' proveniente de região de elevada altitude de Santa Catarina, safra 2016/2017.

Figure 1 - Effect of different temperatures of malolactic fermentation on the color of 'Cabernet Sauvignon' grape from high altitude regions of Santa Catarina, Lages / SC, 2016/2017 harvest.

\section{DISCUSSÃO}

A fermentação malolática é normalmente desejada nos vinhos tintos e brancos de guarda, porque, além de moderar a acidez, proporcionam maior complexidade vinosa. Ao mesmo tempo, não é desejável que ocorra nos tintos jovens e nos brancos frutados, exceto os bem ácidos (AMARANTE, 2010). 
A temperatura é um fator importante na estabilidade das antocianinas porque à medida que se submete a solução de antocianinas a uma temperatura superior à ambiente $\left(25^{\circ} \mathrm{C}\right)$, a sua degradação é maior (STRINGHETA, 1991). o que pode ser observado no presente estudo, qual o aumento da temperatura durante o processo de fermentação malolática, provocou efeitos negativos na coloração dos vinhos Cabernet Sauvignon.

Tanto a intensidade de cor quanto a tonalidade são atributos importantes de um vinho, pois através de seu aspecto são obtidas informações sobre suas qualidades e possíveis defeitos (FREITAS, 2006). A diminuição dos valores de tonalidade corresponde a um aumento mais importante da Abs $520 \mathrm{~nm}$ que mede coloração vermelha em relação a Abs $420 \mathrm{~nm}$ que mede a coloração amarela. De acordo com Somers (1976), as antocianinas apresentam uma elevada absorbância relativa, o que aumenta a Abs 520nm, e, portanto, diminui a tonalidade de cor. As altas temperaturas durante a fermentação malolática provocou aumento da tonalidade de cor, muito em função da redução da coloração vermelha/violácea, a degradação das antocianinas e início do processo oxidativo do vinho Cabernet Sauvignon.

\section{CONCLUSÕES}

A temperatura da fermentação malolática tem influência na composição química do vinho da uva 'Cabernet Sauvignon', sendo que o aumento da temperatura reduz o teor de antocianinas, além de reduzir a coloração vermelha do vinho, representado pelo aumento da tonalidade de coloração dos vinhos. A temperatura da fermentação malolática não influenciou o conteúdo de polifenóis totais do vinho da uva 'Cabernet Sauvignon'. 


\section{REFERÊNCIAS}

AMARANTE, J. O. A. Os Segredos do Vinho. 3. ed. São Paulo: Editora Mescla. 2010. p. $147-153$

BINATI, R.L. Avaliação da fermentação malolática em vinhos de altitude com bactérias ácido-lácticas autóctones selecionadas. Florianópolis, $111 \mathrm{p}$. Dissertação (Mestrado em Biotecnologia e Biociências), - Universidade Federal de Santa Catarina - UFSC, 2015.

BORGHEZAN, M.; VILLAR, L.; SILVA, T.C.; CANTON, M.; GUERRA, M.P.; CAMPOS, C.G.C. Phenology and Vegetative Growth in a New Production Region of Grapevines: Case Study in São Joaquim, Santa Catarina, Southern Brazil. Open Journal of Ecology, v.4, p.321-335, 2014.

FREITAS, D.M. Variação dos compostos fenólicos e de cor dos vinhos de uvas (Vitis vinífera) tintas em diferentes ambientes, Santa Maria, 56 p., Tese (Doutorado): Universidade Federal de Santa Maria, 2006.

JACKSON, R.S. Wine Science: principles and applications. ELSEVIER, 6. ed., 751 p., 2014.

MAKHOTKINA, O.; HERBST-JOHNSTONE, M.; LOGAN, G.; DU TOIDT, W.; KILMARTIN, P.A. Influence of sulfur dioxide additions at harvest on polyphenols, C6compounds and varietal thiols in Sauvignon blanc. American Journal of Enology and Viticulture, v.64, p.203-213, 2013.

MALINOVSKI, L.I.; BRIGHENTI, A.F.; BORGHEZAN, M.; GUERRA, M.P.; FILVA, A.L.; PORRO, D.; STEFANINI, M.; VIEIRA, H.J. Viticultural performance of Italian grapevines in high altitude regions of Santa Catarina State, Brazil. Acta Horticulturae, v.1115, p.203-210, 2016.

MOTA, R.V.; RAMOS, C.L.; PEREGRINO, I.; PURGATTO, E.; SOUZA, C.R.; DIAS, D.R.; REGINA, M.A. Identification of the potential inhibitors os malolatic fermentation in wines. Food Science Techonology, v.38, p.174-179, 2018.

PSZCZOLKOWSKI, P.; LECCO, C.C. Manual de vinificación: Guía práctica para la elaboración de vinos. Universidade Catolica do Chile: Santiago, 2011.

RIZZON, L. A.; MANFROI, L. Fermentação. Embrapa Uva e Vinho, 2006. Disponível em:<https://sistemasdeproducao.cnptia.embrapa.br/FontesHTML/Vinho/SistemaProd ucaoVinhoTinto/fermentacao.htm>, Acesso em: 19 de novembro de 2020.

RIZZON, L.A. Metodologia para análise de vinho. Brasília: Embrapa Informação Tecnológica, 120 p., 2010.

SINGLETON, V.L.; ROSSI, J.A. Colorimetry of total phenolics with phosphomolybdic - phosphotunestic acids reagents. American Journal of Enology and Viticulture, v.16, p.144-158, 1965. 
SOMERS, T.C. Pigment development during ripening of the grapes. Vitis, v.14, p.269277, 1976.

STRINGHETA, P.C. Identificação da estrutura e estudo da estabilidade das antocianinas extraídas da inflorescência de capim gordura (Mellinis minutuflora, Pal de Beauv.), Campinas,138 p. Tese (Doutorado em Ciência e Tecnologia de Alimentos) - UNICAMP, 1991.

WURZ, D.A.; BEM, B.P.; ALLEBRANDT, R.; BONIN, B.; DALMOLIN, G.L.; CANOSSA, A.T.; RUFATO, L.; KRETZSCHMAR, A.A. New wine-growing regions of Brazil and their importance in the evolution of Brazilian wine. BIO Web of Conferences, v.9, p.1-4, 2017. 